\title{
Tunable mechanical coupling between driven microelectromechanical resonators
}

G. J. Verbiest, D. Xu, M. Goldsche, T. Khodkov, S. Barzanjeh, N. von den Driesch, D. Buca, and C. Stampfer

Citation: Appl. Phys. Lett. 109, 143507 (2016); doi: 10.1063/1.4964122

View online: http://dx.doi.org/10.1063/1.4964122

View Table of Contents: http://aip.scitation.org/toc/apl/109/14

Published by the American Institute of Physics 


\title{
Tunable mechanical coupling between driven microelectromechanical resonators
}

\author{
G. J. Verbiest, ${ }^{1}$ D. Xu, ${ }^{1}$ M. Goldsche, ${ }^{1,2}$ T. Khodkov, ${ }^{1,2}$ S. Barzanjeh, ${ }^{3,4}$ N. von den Driesch, ${ }^{2}$ \\ D. Buca, ${ }^{2}$ and C. Stampfer ${ }^{1,2}$ \\ ${ }^{1}$ JARA-FIT and 2 nd Institute of Physics, RWTH Aachen University, 52074 Aachen, Germany \\ ${ }^{2}$ Peter Grünberg Institute (PGI-8/9), Forschungszentrum Jülich, 52425 Jülich, Germany \\ ${ }^{3}$ Institute for Quantum Information, RWTH Aachen University, 52056 Aachen, Germany \\ ${ }^{4}$ Institute of Science and Technology Austria, 3400 Klosterneuburg, Austria
}

(Received 15 July 2016; accepted 20 September 2016; published online 4 October 2016)

\begin{abstract}
We present a microelectromechanical system, in which a silicon beam is attached to a comb-drive actuator, which is used to tune the tension in the silicon beam and thus its resonance frequency. By measuring the resonance frequencies of the system, we show that the comb-drive actuator and the silicon beam behave as two strongly coupled resonators. Interestingly, the effective coupling rate $(\sim 1.5 \mathrm{MHz})$ is tunable with the comb-drive actuator $(+10 \%)$ as well as with a side-gate $(-10 \%)$ placed close to the silicon beam. In contrast, the effective spring constant of the system is insensitive to either of them and changes only by $\pm 0.5 \%$. Finally, we show that the comb-drive actuator can be used to switch between different coupling rates with a frequency of at least $10 \mathrm{kHz}$. Published by AIP Publishing. [http://dx.doi.org/10.1063/1.4964122]
\end{abstract}

Micro- and nanoelectromechanical resonators have attracted much attention - thanks to their potential application as sensors, ${ }^{1-8}$ filters, ${ }^{9-11}$ amplifiers, ${ }^{12,13}$ and logic gates, ${ }^{14}$ with a range of frequencies varying from the $\mathrm{kHz}$ to the GHz. ${ }^{15-17}$ By applying tension, the resonance frequencies can be tuned in-situ. ${ }^{18-20}$ This is usually done by applying a DC voltage to a nearby gate to induce a deformation of the resonator via the electrostatic potential. ${ }^{21-23}$ However, one does not only induce tension by applying an electrostatic potential but also changes the number of charge carriers, ${ }^{24-26}$ which both affect the properties of the resonator. ${ }^{27,28}$ An ideal candidate to circumvent this problem is the comb-drive actuator, which is widely used nowadays to detect the accelerations and rotations as well as to manipulate, stretch, or move objects. $^{29-33}$ By mechanically fixing a resonator to a combdrive, one can purely mechanically induce strain in the resonator. Depending on the type of comb-drive actuator, this induced strain can be either tensile or compressive. The former allows the disentanglement of strain and charge carrier density effects in mechanical resonators, whereas the latter is particularly relevant to gain control over buckling modes in mechanical resonators. ${ }^{34,35}$

Coupled micro- and nanomechanical resonators are known to show sensitive sympathetic oscillation dynamics that show better performance in potential applications than a single resonator. ${ }^{36-40}$ One major challenge for this type of resonators is an in-situ control over the coupling. ${ }^{41}$ From a fundamental point of view, a tunable mechanical coupling is interesting because of the feasibility to coherently manipulate phonon populations in coupled resonators $^{42,43}$ and to create intrinsically localized modes. ${ }^{44}$ From an applied point of view, researchers are able to build novel mass sensors, bandpass filters, and single-electron detectors. $^{45-47}$ Fast in-situ control over the coupling between mechanical resonators is an important step towards mechanical logic gates. ${ }^{48}$ Comb-drives are suspended microelectromechanical systems based on springs, which typically have a resonance frequency in the (low) $\mathrm{kHz}$ range. ${ }^{49-51}$ Once attaching a resonator to this type of micromachine, one could introduce a system of two (strongly) coupled resonators with a potential control of the coupling. Here, we present an electromechanical system consisting of a comb-drive actuator to control the tensile strain in an integrated silicon beam. We show that the device behaves as a system with two strongly coupled resonators, with a coupling rate $\sim 1.5 \mathrm{MHz}$, which is tunable up to $+10 \%$ with the comb-drive actuator as well as down to $-10 \%$ with a side-gate (SG) placed close to the silicon beam.

The devices are fabricated on a substrate consisting of

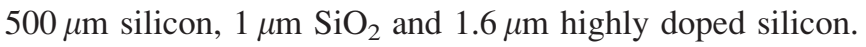
Using the standard e-beam lithography, a chromium hard mask is deposited after which reactive ion etching is used to etch away the highly doped silicon. After removing the hard mask, the $\mathrm{SiO}_{2}$ layer is partly etched away with $10 \%$ hydrofluoric (HF) acid solution to suspend the comb-drive actuator and silicon beam. Finally, a critical point dryer (CPD) is used to suspend the structure and protect it from collapsing to the underlying silicon layer. Fig. 1(a) shows a schematic illustration of the studied device with all its electrical contacts of highly doped silicon as well as the high aspect ratio $\sim 0.156$ in our device. An SEM image of the fabricated device is provided in Fig. 1(b). The fabricated silicon beam is $13.3 \mu \mathrm{m}$ long, $250 \mathrm{~nm}$ wide, and $1.6 \mu \mathrm{m}$ high (Fig. 1(c)). All the measurements were performed using an amplitude modulated down-mixing technique ${ }^{52,53}$ in a variable temperature insert at a temperature of $\sim 2.3 \mathrm{~K}$, which is the base temperature of our home built setup if operated at low $\mathrm{He}$ pressures (1 mbar).

A schematic overview of the electronic measurement circuit is depicted in Fig. 1(d). The capacitors $C_{0}=100 \mathrm{nF}$ and resistors $R_{0}=50 \Omega$ are chosen for impedance matching 

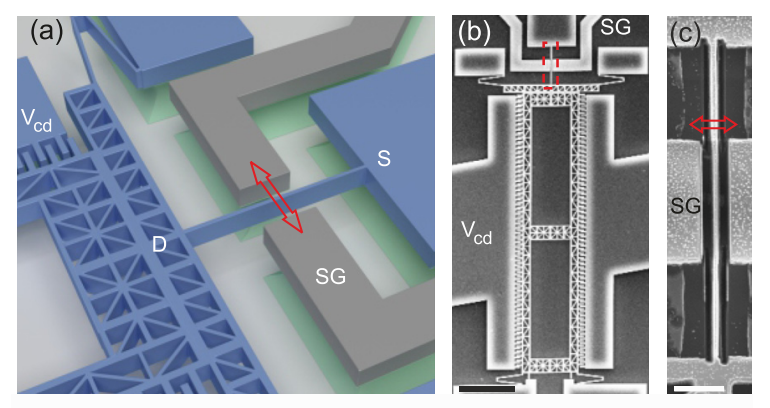

(d)

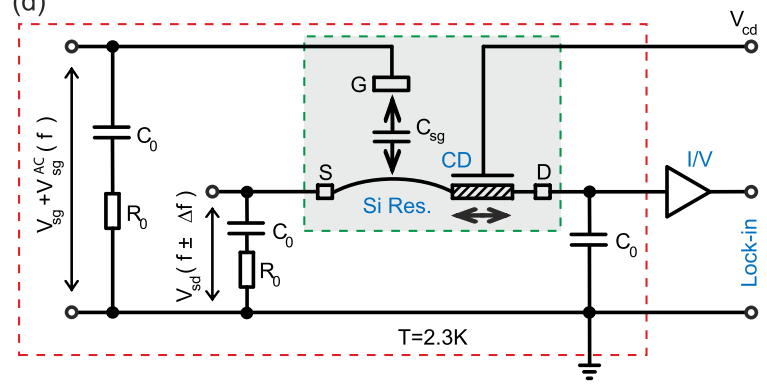

FIG. 1. (a) Schematic illustration of the investigated device. The red arrow indicates the lateral vibration of the silicon beam. Scanning electron microscope (SEM) images of (b) the comb-drive actuator and (c) a zoom in of the dashed red box in panel (b). The scale bars indicate $20 \mu \mathrm{m}$ and $2 \mu \mathrm{m}$, respectively. The electronic circuit depicted in (d) is used to measure the resonance frequency, where the inner green box indicates the device and the red box indicates the printed circuit board (PCB) in the cryostat at $T=2.3 \mathrm{~K}$. An AC signal $\left(V_{s d}(f \pm \Delta f)\right)$ drives the current from source (S) to drain (D) contact, while a sum of DC and AC voltages $\left(V_{s g}+V_{s g}^{A C}(f)\right)$ are applied to the side-gate (SG). The down-mixed current at frequency $\Delta f$ through the resonator is measured by an I/V-converter and a lock-in amplifier. The comb-drive actuator is driven by a DC voltage $V_{c d}$. The capacitors $C_{0}=100 \mathrm{nF}$ and resistors $R_{0}=50 \Omega$ are chosen for impedance matching and decoupling any high frequency signals.

and for decoupling any high frequency signals. The combdrive actuator is actuated capacitively by the DC voltage $V_{c d}$ applied between the comb fingers. To tune the charge carrier density in the silicon beam, a DC voltage $V_{s g}$ is applied to the $\mathrm{SG}$ with a distance of $350 \mathrm{~nm}$ to the silicon beam, which has a capacitive coupling $C_{s g}$. Both $V_{c d}$ and $V_{s g}$ tune the strain in the silicon beam. In addition, an AC voltage $V_{s g}^{A C}$ at frequency $f$ is applied to SG for the capacitive actuation of an out-of-plane mechanical vibration in the silicon beam. This leads to a variation in the conductance of the silicon beam at the same frequency $f$. An AC voltage $V_{s d}$ at frequency $f-\Delta f$ and, simultaneously, at frequency $f+\Delta f$ is applied to the source (S)-drain (D) contacts of the silicon beam. As a consequence, the current through the silicon beam contains a term at frequency $\Delta f$, which is measured by an I/V-converter and a lock-in amplifier. The current at frequency $\Delta f$ comprises two terms: one stems from the mechanical motion of the beam and the other is due to the frequency independent variation in charge carrier density $n$. By including an arbitrary phase $\Delta \phi$ shift between these two terms, the current $I(\Delta f)$ is given by ${ }^{54}$

$$
I(\Delta f)=\left[\frac{\frac{\partial C_{s g}}{\partial z} V_{s g} \frac{z_{0}}{Q} \cos [\beta(f)+\Delta \phi]}{\sqrt{\left(1-\left(\frac{f}{f_{0}}\right)^{2}\right)+\left(\frac{f}{f_{0} Q}\right)^{2}}}+C_{s g} V_{s g}^{A C}\right] 2 \frac{\mathrm{d} G}{\mathrm{~d} n} V_{s d},
$$

where $\beta(f)=\arctan \left(\frac{f_{0}^{2}-f^{2}}{f f_{0} / Q}\right), f_{0}$ and $Q$ are the resonance frequency and its corresponding quality factor, $z_{0}$, is the vibration amplitude of the beam at resonance, $\partial C_{s g} / \partial z$ is the change in capacitive coupling between SG and the silicon beam due to a small beam displacement, and $\mathrm{d} G / \mathrm{d} n$ is the transconductance. Note that the lock-in amplifier measures the absolute total current, which can make a single resonance appear as two peaks in $|I(\Delta f)|$, if the phase $\Delta \phi$ is nonzero. The resonance frequencies of the device are found by sweeping the frequency $f$ while measuring $|I(\Delta f)|$. Typically measured spectra are shown in Figs. 2(a) and 2(b) as a function of $V_{s g}$ at constant $V_{c d}$, and vice versa, respectively (see Sec. II of supplementary material for room temperature measurements). Surprisingly, there are two clear resonant modes. The low-frequency resonant mode in Figs. 2(a) and 2(b) exhibits almost no tunability as a function of $V_{c d}$ or $V_{s g}$, to which we will refer to as the "low sensitive mode" (LSM), whereas the high-frequency resonant mode is at least $\sim 12$ times more sensitive to $V_{c d}$ and $V_{s g}$ and will therefore be called the "highly sensitive mode" (HSM). In contrast, the spectrum obtained on a sample with a fixed comb-drive (see inset Fig. 2(c)) only shows a single resonance frequency.

The resonance frequencies are obtained by fitting Eq. (1) to the raw data (Fig. 2(c)). The extracted resonance frequencies in the dependence of $V_{c d}$ and $V_{s g}$ are shown in Figs. 3(a) and 3(b), respectively. We conclude that tensile strain in the silicon beam induced by the comb-drive actuator via $V_{c d}$ increases the resonance frequency of the HSM, whereas an out-of-plane bending of the silicon beam induced by $V_{s g}$ effectively lowers the resonance frequency. All the
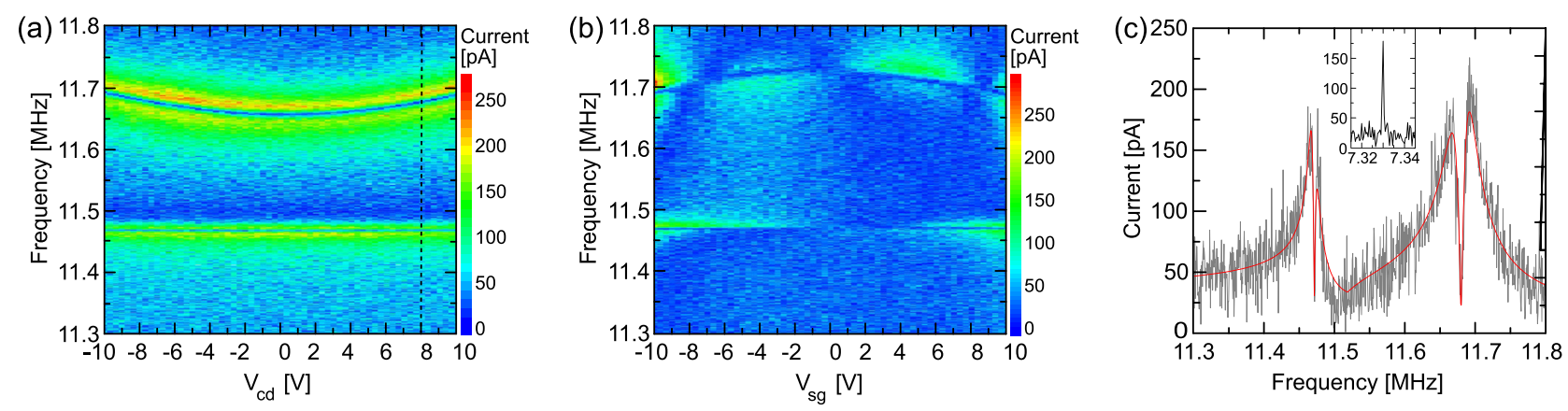

FIG. 2. Measured down-mixed current as functions of (a) the applied $V_{c d}$ at $V_{s g}=10 \mathrm{~V}$ and (b) $V_{s g}$ at $V_{c d}=10 \mathrm{~V}$. The data of the vertically dashed black line in (a) are shown in (c). The red line is the fit to Eq. (1). For comparison, the measured frequency spectrum of a sample with a fixed comb-drive actuator in the inset only shows a single resonance (for more details, see Sec. III of the supplementary material). 

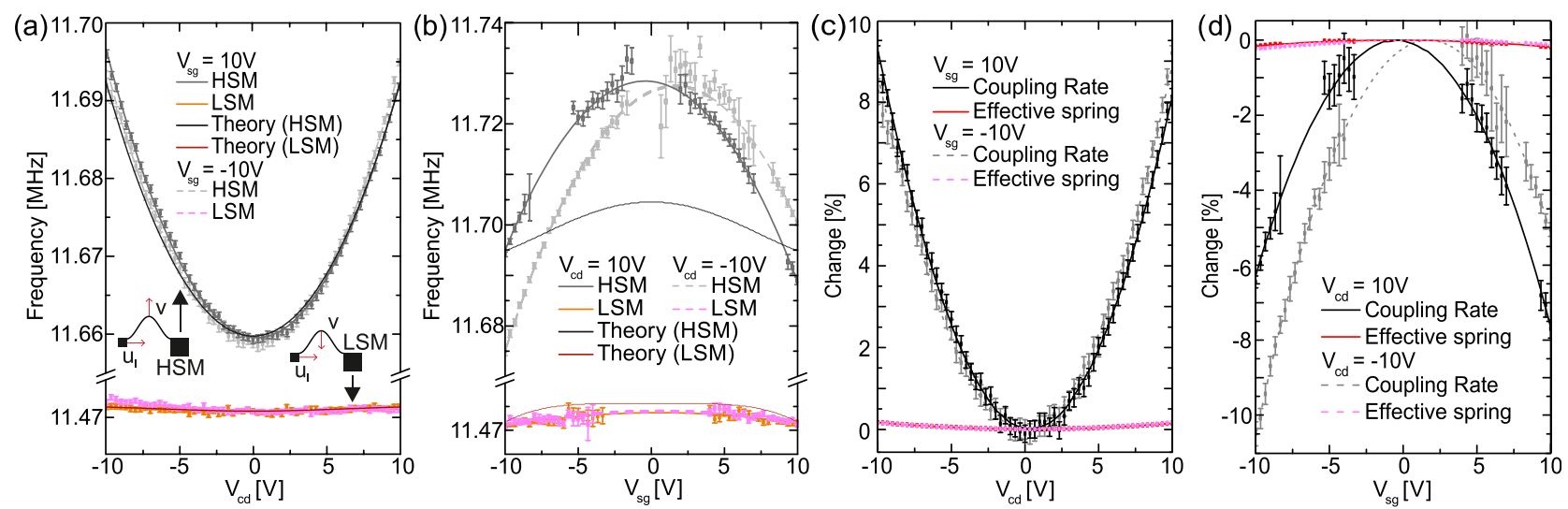

FIG. 3. The extracted resonance frequencies at $\sim 2.3 \mathrm{~K}$ as a function of (a) comb-drive voltage $V_{c d}\left(V_{s g}= \pm 10 \mathrm{~V}\right)$ and (b) SG voltage $V_{s g}\left(V_{c d}= \pm 10 \mathrm{~V}\right)$. The resonance of the HSM corresponds to the silicon beam and the one of the LSM to the comb-drive actuator (see insets in (a) for mode shapes). The theoretically calculated resonance frequencies are also shown as black and red curves. The system of two coupled resonators is characterized by its effective spring and its coupling rate. (c) and (d) show the relative change in these parameters as a function of $V_{c d}$ and $V_{s g}$, respectively. Interestingly, the change in effective spring is below $0.5 \%$, whereas the change in coupling can be as high as $\pm 10 \%$. Moreover, this sign is fully controllable via $V_{c d}$ and $V_{s g}$.

extracted resonance frequencies of the same measurement lie on a quadratic polynomial, as shown with orange and gray solid curves (and pink and light gray dashed curves) in Figs. 3(a) and 3(b). These tunabilities are in agreement with the behavior of the capacitive force between the SG and the silicon beam on the comb-drive actuator, which always scales quadratically with an applied voltage, i.e., $V_{c d}^{2}$ and $V_{s g}^{2}$, corresponding to the strain hardening and the capacitive softening, ${ }^{55}$ respectively. Note that there are slight deviations for the center position at $V_{s g}=0$ (Fig. 3(b)) due to residual charges from the fabrication process or a capacitive crosstalk between the SG and the comb-drive.

The observed two resonant modes in our system are in good agreement with the theoretical prediction. In the theoretical model (see Sec. I of supplementary material), our device is described by a beam attached to a mass, in which a transverse and an axial drive force act on the beam. The beam is doubly clamped in an axial direction and oscillates in the transverse direction, whereas the mass can only move in the axial direction. The resulting dynamics of this system can be expressed by the following equations of motion

$$
\ddot{v}+\lambda_{1} v+\Gamma u_{l}=0, \quad \mu \ddot{u}_{l}+\lambda_{2} u_{l}+\Gamma v=0,
$$

in which $v$ and $u_{l}$ denote the time dependent part of the oscillations of the beam and the mass, respectively, i.e., the silicon beam and the comb-drive. $\lambda_{1}$ and $\lambda_{2}$ describe the normalized effective springs, $\mu$ is the normalized mass ratio between the comb-drive and the silicon beam, and $\Gamma$ indicates the coupling between these two oscillations. All these parameters are related to $V_{c d}$ and $V_{s g}$ via the static bending of the silicon beam and the intrinsic properties of the device: the Young's modulus, the spring constant, the mass density, the geometry and the capacitive couplings. The extracted resonance frequencies are as follows:

$$
\begin{aligned}
& \omega_{1}=\sqrt{\frac{\mu \lambda_{1}+\lambda_{2}-\sqrt{4 \Gamma^{2} \mu+\left(-\mu \lambda_{1}+\lambda_{2}\right)^{2}}}{2 \mu}}, \\
& \omega_{2}=\sqrt{\frac{\mu \lambda_{1}+\lambda_{2}+\sqrt{4 \Gamma^{2} \mu+\left(-\mu \lambda_{1}+\lambda_{2}\right)^{2}}}{2 \mu}} .
\end{aligned}
$$

The frequencies obtained with Eq. (3) are in qualitatively good agreement with the experimental data (blue and red curves in Figs. 3(a) and 3(b)), which allows us to specify the mode type to the HSM and the LSM (see insets in Fig. 3(a) for mode shapes). According to the theory, the HSM depends on the induced tensile strain in the silicon beam with the comb-drive actuator and is therefore attributed to the silicon beam; the LSM does not show a strong dependence on $V_{c d}$ and $V_{s g}$ and represents the resonance of the comb-drive actuator. The fact that these modes are so close together suggests that they are strongly coupled due to the clamping of the silicon beam on the comb-drive actuator. Note that the theory shows quantitatively good agreement with the experiment in Fig. 3(a). However, it does not describe the experimental SG voltage dependence very well (see Fig. 3(b)), which is due to the following approximations in the theory: the transverse force acts on the entire length of the beam and the neglected capacitive softening.

In the case of a very similar device but with fixed combdrive (see Sec. III of supplementary material), we observe only a single resonant mode (see inset Fig. 2(c)). We conclude that the comb-drive is a second mechanical degree of freedom and thus induces a second mode. In addition, the quality factor in this case is $\sim 10000$ which is approximately 10 to 100 times larger than the quality factor of the device, where the comb-drive actuator is movable (see Sec. IV of supplementary material). This suggests that a significant amount of energy is transferred from the silicon beam into the comb-drive actuator and vice versa.

To investigate the actual effective spring and effective coupling rate, the system of two strongly coupled resonators is described by the following system of equations:

$$
\begin{aligned}
& (2 \pi)^{-2} \omega^{2} x_{1}+\lambda^{2} x_{1}+\gamma^{2} x_{2}=0, \\
& (2 \pi)^{-2} \omega^{2} x_{2}+\lambda^{2} x_{2}+\gamma^{2} x_{1}=0,
\end{aligned}
$$

where $x_{1}$ and $x_{2}$ are effective displacements. The solutions are the two resonance frequencies $\omega_{1}$ and $\omega_{2}$ defined by Eq. (3). Note that $\lambda^{2}=0.5\left(\omega_{1}^{2}+\omega_{2}^{2}\right)(2 \pi)^{-2}$ is the (squared) effective spring of the system and $\gamma^{2}=0.5\left|\omega_{1}^{2}-\omega_{2}^{2}\right|(2 \pi)^{-2}$ is the (squared) effective coupling rate, which are listed in Table I for specific $V_{c d}$ and $V_{s g}$. Surprisingly, the extracted 
TABLE I. The absolute values of the coupling rates and effective springs for different $V_{c d}$ and $V_{s g}$.

\begin{tabular}{lccccc}
\hline \hline & \multicolumn{2}{c}{$V_{s g}=10 \mathrm{~V}$} & & \multicolumn{2}{c}{$V_{c d}=10 \mathrm{~V}$} \\
\cline { 2 - 3 } \cline { 5 - 6 } & $V_{c d}=10 \mathrm{~V}$ & $V_{c d}=-10 \mathrm{~V}$ & & $V_{s g}=10 \mathrm{~V}$ & $V_{s g}=-10 \mathrm{~V}$ \\
\hline $\begin{array}{l}\text { Coupling rate } \\
\text { (MHz) }\end{array}$ & 1.72 & 1.75 & & 1.48 & 1.47 \\
$\begin{array}{l}\text { Effective spring } \\
(\mathrm{MHz})\end{array}$ & 11.60 & 11.60 & & 11.57 & 11.57 \\
\hline \hline
\end{tabular}

effective spring and coupling rate show completely different behavior (see Figs. 3(c) and 3(d)). The effective spring $\lambda$ is only weakly dependent on both $V_{c d}$ and $V_{s g}$. A maximum change of only $0.5 \%$ in $\lambda$ is observed in our measurements, which could be attributed to a large difference in the masses of the two oscillating parts. In contrast, the effective coupling rate $\gamma$ can be increased up to $10 \%$ by applying tensile strain through $V_{c d}$, or it can be decreased down with $10 \%$ by applying an out-of-plane bending through $V_{s g}$. Therefore, our device geometry gives us an independent knob to control the coupling between the two resonators.

As the effective coupling rate $\gamma$ is tunable over $10 \%$ with the comb-drive actuator, $V_{c d}$ is a control voltage to switch between different coupling rates. Theoretically, it is even possible to fast switch the coupling between the two resonators, showing the feasibility of mechanical logic gates. To show that the comb-drive actuator works as a switch, we applied square pulses with a frequency of $10 \mathrm{kHz}$ to the comb-drive actuator (see Fig. 4). The period of this frequency was chosen far above the response time of the combdrive actuator and the silicon beam, which were estimated from the resonance frequency and its quality factor to be $36 \mu$ s and $12 \mu \mathrm{s}$, respectively. For large square pulse amplitudes, the resonance frequency indeed splits into two, which shows the possibility to switch the coupling rate between the two resonators. Here, the coupling rate is switched between $1.39 \mathrm{MHz}$ and $1.61 \mathrm{MHz}$ at the pulse amplitude $5 \mathrm{~V}$.

In conclusion, we presented an electromechanical system, in which a silicon beam was attached to a comb-drive actuator to control the tension that operates at cryogenic temperatures. By measuring the resonance frequencies of the system, we showed that the device behaves as two strongly

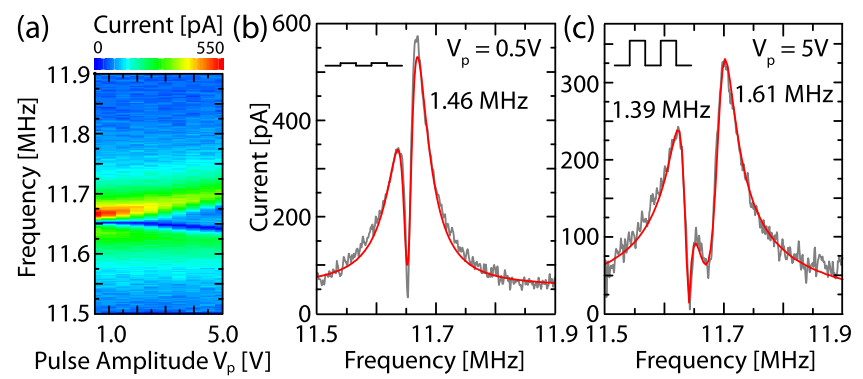

FIG. 4. (a) The down mixed current as a function of the square wave amplitude send through the comb-drive actuator. The period of the square wave $(10 \mathrm{kHz})$ is far above the response time of the observed resonances. Therefore, for large enough square wave amplitude, the resonance frequency splits into two (shown in (b) and (c) with coupling rates), showing the possibility to switch the coupling rate between the two resonators almost instantaneously. coupled resonators. Surprisingly, the effective coupling rate $(\sim 1.5 \mathrm{MHz})$ is tunable with the comb-drive actuator $(+10 \%)$ as well as with a side-gate $(-10 \%)$ placed close to the silicon beam. In contrast, the effective spring constant of the system is insensitive to an applied voltage to either the comb-drive actuator or the side-gate. From an applied point of view, the high tunability of coupling rate would be very interesting if two resonators are attached to the comb-drive actuator as multifunctional force sensors. Finally, we showed that the comb-drive actuator can be used to switch between different coupling rates with a frequency of at least $10 \mathrm{kHz}$, which is particularly interesting for the future mechanical logic devices, in which it is necessary to fast switch between the two states.

See supplementary material for the full theoretical description of the mechanical system, the room temperature measurements, the measurements on a device with a fixed comb-drive actuator, and the quality factors.

We acknowledge the support from the Helmholtz Nanoelectronic Facility (HNF) and funding from the ERC (GA-Nr. 280140).

${ }^{1}$ D. Culler, D. Estrin, and M. Srivastava, Computer 37, 41 (2004).

${ }^{2} \mathrm{M}$. Mori, "Fast response micro-safing sensor for air bag systems," SAE Technical Paper 1999-01-0758, 1999.

${ }^{3}$ F. Tabak, E. Disseldorp, G. Wortel, A. Katan, M. Hesselberth, T. Oosterkamp, J. Frenken, and W. van Spengen, Ultramicroscopy 110, 599 (2010).

${ }^{4}$ E. Disseldorp, F. Tabak, A. Katan, M. Hesselberth, T. Oosterkamp, J. Frenken, and W. van Spengen, Rev. Sci. Instrum. 81, 043702 (2010).

${ }^{5}$ C. Martin-Olmos, H. Rasool, B. Weiller, and J. Gimzewski, ACS Nano 7, 4164 (2013).

${ }^{6}$ K. Schwab and M. Roukes, Phys. Today 58(7), 36 (2005).

${ }^{7}$ M. Wallquist, K. Haetermmerer, P. Rabl, M. Lukin, and P. Zoller, Phys. Scr. 2009, 014001.

${ }^{8}$ J. Eisert, M. Plenio, S. Bose, and J. Hartley, Phys. Rev. Lett. 93, 190402 (2004).

${ }^{9}$ L. Yang, T. Huang, and P. Chang, Sens. Actuators, A 90, 148 (2001).

${ }^{10}$ V. Gouttenoire, T. Barois, S. Perisanu, J.-L. Leclercq, S. T. Purcell, P. Vincent, and A. Ayari, Small 6, 1060 (2010).

${ }^{11}$ B. Piekarski, D. DeVoe, M. Dubey, R. Kaul, and J. Conrad, Sens. Actuators, A 91, 313 (2001).

${ }^{12}$ G. Rebeiz, RF MEMS: Theory, Design, and Technology (John Wiley \& Sons, 2004).

${ }^{13}$ R. Karabalin, R. Lifshitz, M. Cross, M. Matheny, S. Masmanidis, and M. Roukes, Phys. Rev. Lett. 106, 094102 (2011).

${ }^{14}$ C. Tsai, W. Kuo, C. Lin, and T. Chen, J. Micromech. Microeng. 18, 045001 (2008).

${ }^{15}$ A. Cleland and M. Roukes, Appl. Phys. Lett. 69, 2653 (1996).

${ }^{16}$ C. Nguyen, V. Gutnik, and R. Howe, U.S. patent 5,839,062 (17 November 1998).

${ }^{17}$ K. Ekinci, Y. Yang, X. Huang, and M. Roukes, Appl. Phys. Lett. 81, 2253 (2002).

${ }^{18}$ E. Buks and M. L. Roukes, J. Microelectromech. Syst. 11, 802 (2002).

${ }^{19}$ D. Zhu, M. J. Tudor, and S. P. Beeby, Meas. Sci. Technol. 21, 022001 (2010).

${ }^{20}$ V. R. Challa, M. Prasad, Y. Shi, and F. T. Fisher, Smart Mater. Struct. 17, 015035 (2008).

${ }^{21}$ Y. Lei, J. Sun, and X. Gong, Physica B 461, 61 (2015).

${ }^{22}$ S. Y. No, A. Hashimura, S. Pourkamali, and F. Ayazi, in Proceedings of the Solid-State Sensor, Actuator and Microsystems Workshop, Hilton Head (Citeseer, 2002), pp. 281-284.

${ }^{23}$ G. Piazza, R. Abdolvand, G. K. Ho, and F. Ayazi, Sens. Actuators, A: Phys. 111, 71 (2004).

${ }^{24}$ B. Lassagne, D. Garcia-Sanchez, A. Aguasca, and A. Bachtold, Nano Lett. 8, 3735 (2008). 
${ }^{25}$ D. Grogg, M. Mazza, D. Tsamados, and A. M. Ionescu, in 2008 Electron Devices Meeting (IEEE, 2008).

${ }^{26}$ Y. Sun, S. Thompson, and T. Nishida, J. Appl. Phys. 101, 104503 (2007).

${ }^{27}$ D. Li, Y. Wu, P. Kim, L. Shi, P. Yang, and A. Majumdar, Appl. Phys. Lett. 83, 2934 (2003).

${ }^{28}$ R. Lifshitz and M. L. Roukes, Phys. Rev. B 61, 5600 (2000).

${ }^{29}$ W. Tang, T. Nguyen, M. Judy, and R. Howe, Sens. Actuators, A: Phys. 21, 328 (1990).

${ }^{30}$ L. Lin, C. Nguyen, R. Howe, and A. Pisano, "Micro electromechanical filters for signal processing," in 5th IEEE Micro Electro Mechanical Systems Workshop (MEMS 92), Travemuende, Germany, 4-7 February 1992 (IEEE, 1992), pp. 226-231.

${ }^{31}$ S. Su, H. Yang, and A. Agogino, IEEE Sens. J. 5, 1214 (2005).

${ }^{32}$ A. P. Lee and A. P. Pisano, J. Microelectromech. Syst. 1, 70 (1992).

${ }^{33}$ E. J. Garcia and J. J. Sniegowski, in Proceedings of the 8th International Conference on Solid-State Sensors and Actuators and Eurosensors IX, Transducers' 95 (IEEE, 1995), Vol. 1, pp. 365-368.

${ }^{34}$ M. Saif, J. Microelectromech. Syst. 9, 157 (2000).

${ }^{35}$ M. Sulfridge, T. Saif, N. Miller, and M. Meinhart, J. Microelectromech. Syst. 13, 725 (2004).

${ }^{36}$ M. Spletzer, A. Raman, A. Q. Wu, X. Xu, and R. Reifenberger, Appl. Phys. Lett. 88, 254102 (2006).

${ }^{37}$ H. Okamoto, N. Kitajima, K. Onomitsu, R. Kometani, S.-I. Warisawa, S. Ishihara, and H. Yamaguchi, Appl. Phys. Lett. 98, 014103 (2011).

${ }^{38}$ S.-B. Shim, M. Imboden, and P. Mohanty, Science 316, 95 (2007).

${ }^{39}$ R. Karabalin, M. Cross, and M. Roukes, Phys. Rev. B 79, 165309 (2009).
${ }^{40}$ T. Faust, J. Rieger, M. J. Seitner, P. Krenn, J. P. Kotthaus, and E. M. Weig, Phys. Rev. Lett. 109, 037205 (2012).

${ }^{41}$ S. C. Masmanidis, R. B. Karabalin, I. De Vlaminck, G. Borghs, M. R. Freeman, and M. L. Roukes, Science 317, 780 (2007).

${ }^{42}$ H. Okamoto, A. Gourgout, C.-Y. Chang, K. Onomitsu, I. Mahboob, E. Y. Chang, and H. Yamaguchi, Nat. Phys. 9, 480 (2013).

${ }^{43}$ S. Barzanjeh and D. Vitali, Phys. Rev. A 93, 033846 (2016).

${ }^{44}$ M. Sato, B. Hubbard, A. Sievers, B. Ilic, D. Czaplewski, and H. Craighead, Phys. Rev. Lett. 90, 044102 (2003).

${ }^{45}$ M. Spletzer, A. Raman, H. Sumali, and J. P. Sullivan, Appl. Phys. Lett. 92, 114102 (2008).

${ }^{46}$ H. Okamoto, T. Kamada, K. Onomitsu, I. Mahboob, and H. Yamaguchi, Appl. Phys. Express 2, 062202 (2009).

${ }^{47}$ S. Pourkamali and F. Ayazi, Sens. Actuators, A 122, 307 (2005).

${ }^{48}$ I. Mahboob and H. Yamaguchi, Nat. Nanotechnol. 3, 275 (2008).

${ }^{49}$ P. R. Patterson, D. Hah, H. Nguyen, H. Toshiyoshi, R.-M. Chao, and M. C. Wu, in Proceedings of the Fifteenth IEEE International Conference on Micro Electro Mechanical Systems (IEEE, 2002), pp. 544-547.

${ }^{50}$ R. Legtenberg, A. Groeneveld, and M. Elwenspoek, J. Micromech. Microeng. 6, 320 (1996).

${ }^{51}$ Y. Sun, S. N. Fry, D. Potasek, D. J. Bell, and B. J. Nelson, J. Microelectromech. Syst. 14, 4 (2005).

${ }^{52}$ R. Knobel, C. Yung, and A. Cleland, Appl. Phys. Lett. 81, 532 (2002).

${ }^{53}$ V. Sazonova, Y. Yaish, H. Üstünel, D. Roundy, T. A. Arias, and P. McEuen, Nature 431, 284 (2004).

${ }^{54}$ V. Sazonova, Ph.D. thesis, Cornell University, 2006.

${ }^{55}$ R. Lifshitz and M. Cross, Nonlinear Dynamics of Nanosystems (WileyVCH Verlag GmbH \& Co. KGaA, 2010), pp. 221-266. 